

\title{
Deep art-science collaborations: the mother of invention
}

\author{
Caroline Wellbery MD
}

\begin{abstract}
Dept. of Family Medicine, PreClinical Sciences, Georgetown University Medical Center 3900 Reservoir Road, Washington, DC 20007, wellberc@georgetown.edu_
\end{abstract}

\begin{abstract}
Art-science collaborations organize interdisciplinary inquiries around research themes of mutual interest. The clash between art's open-endedness, and its mortal enemy, the hypothesis-driven protocol, make it difficult for scientists to understand how art-science collaborations can be mutually beneficial. As it turns out, the boundary-challenging aspects of these collaborations often force participants to question the context of their research and their discipline's internal culture. Deep collaborations, especially those aided by technological enhancements, could lead to a sort of creative hypothesis-generating ambiance among participants. Art-science collaborations will and should continue to proliferate as we enter a yet another renaissance showing that interdisciplinary cross-pollination is the mother of invention.
\end{abstract}

Art-science collaborations are flourishing these days. Artists are flooding into scientific laboratories. Interdisciplinary groups comprising scientists, engineers and artists are exploring innovative approaches to old problems. Academic think tanks and journals such as MIT's Medialab and Leonardo give countless examples of artscience partnerships. What are art-science collaborations? Simply put, these projects organize interdisciplinary inquiries around research themes of mutual interest. (1) As a cultural phenomenon, the recent popularity of art-science collaborations seems to derive from the perception in the workplace that collaborative teams do better than individual workers by tapping into the superior intelligence of the collective mind and improving productivity. But our global and technological interconnectivity may also be playing a role by blurring disciplinary boundaries.

These explanations for the proliferation of the art-science community raise the question of what these interdisciplinary projects can achieve. Methodologically, science is tied to a relatively fixed procedural template. As Dr. Julian Rayner, a malaria scientist participating in an art-science collaboration noted in an interview (2) discussing the role of art produced in his lab, "I don't think you could say how it's influenced the sort of experiments we do. I don't think you could point to the protocols or the direction of our research and say this has changed this." Science is fundamentally directional, driven by questions that can be proven or disproven. Art, 
by contrast, is an open-ended dalliance. Even in a clinically-focused art-science collaboration that a group of us are currently discussing as part of a multiinstitutional planning grant, open-endedness is a core feature of the methodology. In our proposed collaboration targeting the quality of patients' hospital experience, artists will be sent into the hospital setting to observe protocols and practices. Even though our ultimate goal is to devise an intervention that would improve the hospital experience for physicians, patients and staff, our project investigators have determined that it would be counter-productive to script the artists' gaze in any way that would hamper their imaginative response to what they observe. To honor this question-generating open-endedness, we have put the generation of testable outputs on hold, reserving them for future expansions of this initial project.

The clash between open-endedness, and its mortal enemy, the hypothesis-driven protocol, make it difficult for scientists to understand how art-science collaborations can be mutually beneficial. As previously mentioned, Julian Rayner, the malaria researcher, resisted the idea of any scientific value accruing from the artist's presence. To be sure, art-inspired serendipitous discoveries, such as the origami-based inspiration for the development of a cardiac stent (3), can find fertile soil in the shared creativity of scientists and artists working together. But most of the results of art-science collaborations thus far are more subtle and difficult to quantify. The immediate benefits of introducing different perspectives into artscience collaborations are both more predictable and less spectacular than any hoped-for ground-breaking discoveries. The New York-based Art\&Science website states that its purpose is "to raise public awareness about artists and scientists using science and technology to explore new forms of creative expression, and to increase communication and collaboration between these fields." (4)

This boundary-challenging communicative benefit should be celebrated as a crucial sine qua non of interdisciplinary collaborations. For example, Deborah Robinson, the artist who collaborated with Julian Rayner, was surprised to discover how insular the research laboratory could be. Just at a point in her career when she herself was ready to expand her professional horizons by opening her work up to social and historical influences, she found the opposite circumstance in the malaria lab. As Robinson said: "I became really interested in the fact that in that lab of about 28 researchers in the two teams very few of them had ever visited Africa. They were like lab animals: they didn't think outside and the more I looked at malaria I realized it can't be extricated from geopolitical issues. And that was a turning point and I began to look outside of the lab."

Robinson had obtained a Wellcome Trust grant to join Rayner's malaria lab. She spent three weeks with researchers Julian Rayner and Oliver Billker, growing parasites, smearing blood samples, visiting the insectory, seeing how the researchers did their work, and spending time talking to them about their process, all in the spirit of information-gathering. Her final product, an artistic interpretation of malaria eradication programs had subtle but incendiary effects on the research community, forcing them to question the context of their research and the 
laboratory's internal culture. In particular, as Rayner said, the presence of outsiders in the lab challenges the scientist to reframe his or her parochial perspective:

The impact of work like this on people's lives and understanding and that sort of thing is much less quantifiable but I would say just as important. When students come through my lab, there's this sort of output: they get a $\mathrm{PhD}$ and they publish some nice papers - that's a sort of quantitative output. But there's also the more interesting, subtler output how the time in your lab has changed the way they do science and they think about science and their attitude to collaboration and all these other less quantifiable acts which we never really deal with but that are equally if not more important than the letters after their name and the papers that they publish.

Similarly, Amar Dhand, a neuroscientist working with a textile artist on stroke patients' social networks, found himself compelled to think more about his patients' immediate and practical needs through his collaborative work with artist Lindsay Obermeyer, resulting precisely from this different perspective. (5) The questions she asked at his meetings made his team uncomfortable. The researchers felt their research was far too preliminary to translate into the sort of patient-oriented demands Obermeyer was making, pushing them to think about applications of their work to patient care. "The artist-patient," Dhand said "...forces the scientist to think about social impact and social application," even while the researchers feel that such applications are premature.

This difference in the perspective of artists and scientists suggests that more enduring, intensive collaborations could challenge the current art-science construct which positions artists hierarchically as commentators of scientific work without changing it. Deep collaborations could lead to a sort of creative hypothesisgenerating ambiance among participants. Historically, artists such as Dürer who crafted meticulous celestial maps and animal drawings, gave scientists new templates for imaging their work. (6) The angst Lindsey Obermeyer and Deborah Robinson invited with their questions and observations may become stimulating provocations for scientists to think differently about their work. (7)

In effecting a paradigmatic change, art-science collaborations may receive help from an unlikely quarter. It is clear that the boundaries between art and science are becoming increasingly fluid through the mediating effect of technology. Tangible outcomes are particularly likely in medicine, which straddles the human relational and bioscientific domains. Thus, technologically-driven inventions such as George Khut's interactive digital device to alleviate pain in children, in a variant of biofeedback, integrate art, play, technology and positive medically relevant impact. (8) Art-science collaborations will and should continue to proliferate as we enter a yet another renaissance showing that interdisciplinary cross-pollination is the mother of invention.

References 
1. Caroline Wellbery, C. Art-Science Collaborations - Avenues toward Medical Innovation. N Engl J Med 2015; 373:2495-2497.

2. Based on interviews with Julian Rayner (March 22, 2016) and Deborah Robinson (March 26, 2016)

3. Peraza-Hernandez EA, Hartl DJ, Malak RJ, Lagoudas DC. Origami-inspired active structures: a synthesis and review. Smart Mater Struct 2014;23(9):094001

4. http://www.asci.org/artikel384.html Accessed June11, 2016

5. Based on an interviews with Amar Dhand (April 2, 2016)

6. "When art advanced science" Harvard News. http://news.harvard.edu/gazette/story/2011/12/when-art-advanced-science/ Accessed June11, 2016

7. http://blogs.plos.org/attheinterface/2013/06/19/why-art-and-science/ Accessed June11, 2016

8. George Khut, Bright Hearts. http://georgekhut.com/2011/11/brightheartsprototypes-at-uts-dab-lab/\#4 Accessed June11, 2016 